\title{
Heavy flavour electroweak physics at LEP1 and SLD
}

\author{
R. Hawkings ${ }^{\text {a }}$

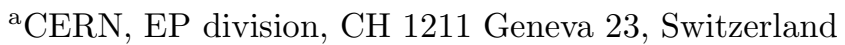

Heavy flavour electroweak measurements from LEP1 and SLD are discussed, with an emphasis on the latest b quark asymmetry results from OPAL and DELPHI. The heavy flavour electroweak fit results are interpreted in terms of coupling parameters and the effective electroweak mixing angle $\sin ^{2} \theta_{\mathrm{W}}^{\text {eff }}$.

\section{Introduction}

Measurements of the couplings of the $\mathrm{Z}^{0}$ to heavy quarks (b and c) provide stringent tests of the Standard Model and allow parameters of the theory (for example the top and Higgs masses) to be constrained via their influence on radiative corrections. The fraction of hadronic $\mathrm{Z}^{0}$ decays to b quarks $(\Gamma(\mathrm{b} \overline{\mathrm{b}}) / \Gamma$ (hadrons $\left.) \approx R_{\mathrm{b}}\right)$ is sensitive to vertex corrections involving top quarks and $\mathrm{W}$ bosons, whilst the parity violating couplings of the $\mathrm{Z}^{0}$ to fermions results in an asymmetry $A_{\mathrm{FB}}^{\mathrm{b}}$ in the differential cross-section for $b$ quark production:

$\frac{\mathrm{d} \sigma}{\mathrm{d} \cos \theta} \propto 1+\cos ^{2} \theta+\frac{8}{3} A_{\mathrm{FB}}^{\mathrm{b}} \cos \theta$,

where $\theta$ is the angle between the directions of the incoming electron and outgoing b quark, and where initial and final state radiation, quark mass and higher order terms have been neglected. At the $\mathrm{Z}^{0}$ pole, the asymmetry $A_{\mathrm{FB}}^{\mathrm{b}, 0}$ is given by $A_{\mathrm{FB}}^{\mathrm{b}, 0}=\frac{3}{4} A_{\mathrm{e}} A_{\mathrm{b}}$ where the coupling parameters of the $\mathrm{Z}^{0}$ to electrons and b quarks, $A_{\mathrm{e}}$ and $A_{\mathrm{b}}$ depend on the effective vector and axial vector couplings of the $\mathrm{Z}^{0}$. Within the context of the Standard Model, these depend on the effective electroweak mixing angle $\sin ^{2} \theta_{\mathrm{W}}^{\text {eff }}$ and are sensitive to the unknown Higgs mass. At SLD, the high electron beam polarisation of the SLC linear collider allows the measurement of asymmetries separately for left- and right-polarised electrons. The resulting 'left-right-forward-backward asymmetry' is directly sensitive to the quark coupling $A_{\mathrm{b}}$ or $A_{\mathrm{c}}$, rather than the product of quark and electron couplings measured at LEP.
The measurements of $R_{\mathrm{b}}$ and $R_{\mathrm{c}}(\approx$ $\Gamma(c \overline{\mathrm{c}}) / \Gamma$ (hadrons) have been stable for some time, and no new results were presented. However, two new asymmetry measurements were submitted to this conference, namely a measurement of the b quark asymmetry using an inclusive tag from OPAL [1], and a simultaneous measurement of the $\mathrm{b}$ and $\mathrm{c}$ quark asymmetries using semileptonic decays from DELPHI [2]. These measurements are discussed in more detail below, before a presentation and interpretation of the fit to all available heavy flavour electroweak data.

\section{New analyses}

The new OPAL b quark asymmetry analysis improves on a previous analysis by using a more efficient and pure b tag developed for the $R_{\mathrm{b}}$ measurement, b quark charge tagging developed for $\mathrm{B}^{0}$ oscillation and $\mathrm{CP}$ violation measurements, an increased $\cos \theta$ acceptance and a more sophisticated fit procedure. Approximately $0.5 \dot{\mathrm{M}}$ hadronic $\mathrm{Z}^{0}$ decays recorded primarily for calibration purposes during the LEP2 era were also added to the $4 \mathrm{M}$ events from LEP1.

Like other inclusive b asymmetry measurements, the OPAL analysis exploits the structure of $\mathrm{Z}^{0} \rightarrow \mathrm{b} \overline{\mathrm{b}}$ events with two back-to-back jets to measure almost all the required event properties from the data themselves. Hemispheres in $\mathrm{Z}^{0} \rightarrow \mathrm{b} \overline{\mathrm{b}}$ events are tagged using a combination of secondary vertex and lepton information, and the tagging efficiency for $\mathrm{b}$ and $\mathrm{c}$ quarks determined from ratios of single and double tagged events. The b quark charge in each hemisphere is deter- 
mined using a combination of jet charges (a sum of all track charges in each jet, weighted according to the momentum of each track), secondary vertex charges and charged kaon information, exploiting the decay chain $\mathrm{b} \rightarrow \mathrm{c} \rightarrow \mathrm{s} \rightarrow \mathrm{K}^{-}$. The fraction of events in which the charge determinations in both hemispheres agree is used to measure the reliability of the charge determination (and hence the dilution of the true b quark asymmetry) directly from the data. The b quark asymmetry is then extracted in 70 analysis bins of differing b purity and $\cos \theta$, and the results combined. The final result [1] has an uncertainty of just over half that of the previous OPAL inclusive result [3].

The new DELPHI analysis [2] measures both the $\mathrm{b}$ and $\mathrm{c}$ quark forward-backward asymmetries using the charge of leptons produced in semileptonic $\mathrm{b} \rightarrow \ell^{-}$and $\mathrm{c} \rightarrow \ell^{+}$decays. The two sources of leptons are separated from 'cascade' decays ( $\mathrm{b} \rightarrow \mathrm{c} \rightarrow \ell^{+}$, which gives the 'wrong' lepton charge) and background using a neural network exploiting lepton momentum, jet shape, lifetime and opposite hemisphere charge information. The latter is used to control the dilution due to $\mathrm{B}-\overline{\mathrm{B}}$ mixing, which can also be measured using the fraction of events with two leptons of the same charge. This analysis updates previous DELPHI results using a more sophisticated fitting procedure, and is now close to finalisation.

\section{Heavy flavour electroweak fit}

The experimental measurements of the heavy flavour partial widths and asymmetries are correlated, both due to direct dependencies of the measurement of one parameter on the assumed value of another, and because of common external inputs (including gluon splitting rates, bottom and charm hadron fragmentation functions, lifetimes and decay branching ratios). The values of the partial widths and asymmetries are therefore extracted using a simultaneous fit to all electroweak data, taking into account correlations and common systematic uncertainties. Several auxiliary parameters are also determined in this fit, including charm hadron production fractions, and b semileptonic branching ratios [4]. The asymme- tries measured at LEP ( $A_{\mathrm{FB}}^{\mathrm{b}}$ and $\left.A_{\mathrm{FB}}^{\mathrm{c}}\right)$ and SLD $\left(A_{\mathrm{b}}\right.$ and $\left.A_{\mathrm{c}}\right)$ are treated as independent parameters.

In a first fit, the LEP asymmetries measured both on and off $( \pm 2 \mathrm{GeV})$ the $\mathrm{Z}^{0}$ peak are treated separately. Since the resulting variations with centre-of-mass energy are consistent with the Standard Model expectation, the measurements are all translated to the $\mathrm{Z}^{0}$ peak, corrected for photon radiation and quark mass effects and combined. The resulting measurements of the pole asymmetries are:

$A_{\mathrm{FB}}^{\mathrm{b}, 0}=0.0995 \pm 0.0015 \pm 0.0007$

$A_{\mathrm{FB}}^{\mathrm{c}, 0}=0.0713 \pm 0.0031 \pm 0.0018$

where the first error is statistical and the second systematic in each case. The individual measurements of $A_{\mathrm{FB}}^{\mathrm{b}, 0}[1,2,5,6]$ are shown in Figure 1 , and the combined result is dominated by the inclusive jet charge analyses from ALEPH, DELPHI and OPAL. The final uncertainty is dominated by data statistics, and the common experimental systematic uncertainty is only 0.0004 , resulting mainly from the understanding of corrections due to gluon radiation.

The individual measurements of $A_{\mathrm{FB}}^{\mathrm{c}, 0}[2,6,7]$ are shown in Figure 2. The measurements using leptons and $\mathrm{D}^{*}$ mesons are consistent, providing confidence that the flavour separation between semileptonic $b$ and $c$ decays is well understood. Again, the uncertainty is statistics dominated, and the common systematic uncertainty is 0.0009 .

The combined results for the partial widths are

$$
\begin{aligned}
& R_{\mathrm{b}}=0.21644 \pm 0.00065 \\
& R_{\mathrm{c}}=0.1718 \pm 0.0031
\end{aligned}
$$

based entirely on final published analyses [8]. These results are compatible with the expectation from the Standard Model and the direct top mass measurement from the Tevatron.

\section{Interpretation of asymmetry results}

The measurements of $A_{\mathrm{FB}}^{\mathrm{b}, 0}$ from LEP, and $A_{\mathrm{b}}$ from SLD are shown in Figure 3, together with the measurement of $A_{\mathrm{e}}$ from the inclusive left- 


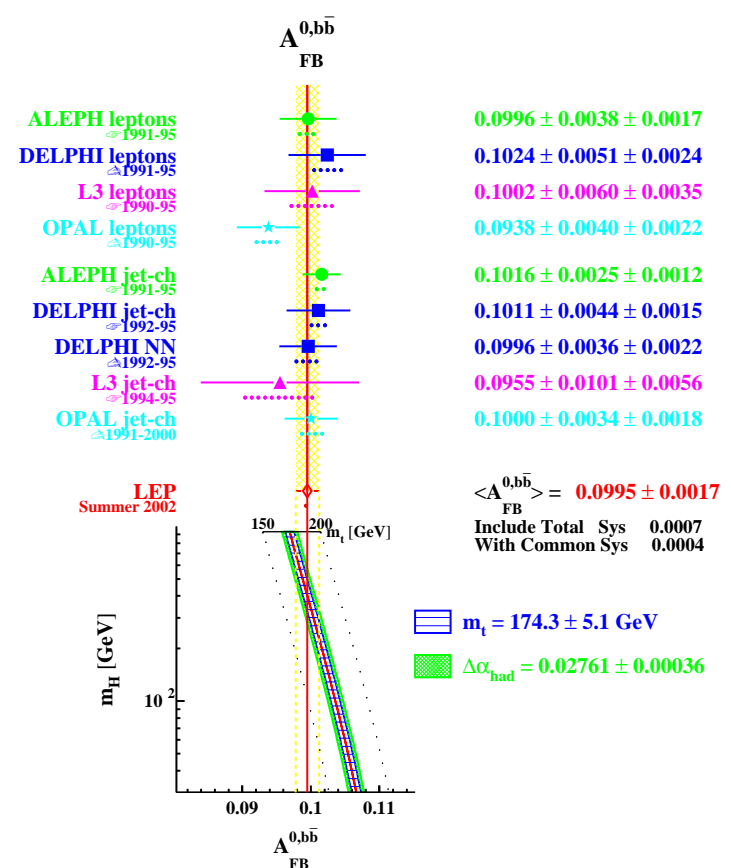

Figure 1. Measurements of $A_{\mathrm{FB}}^{\mathrm{b}, 0}$, the $\mathrm{b}$ quark asymmetry at the $\mathrm{Z}^{0}$ pole.

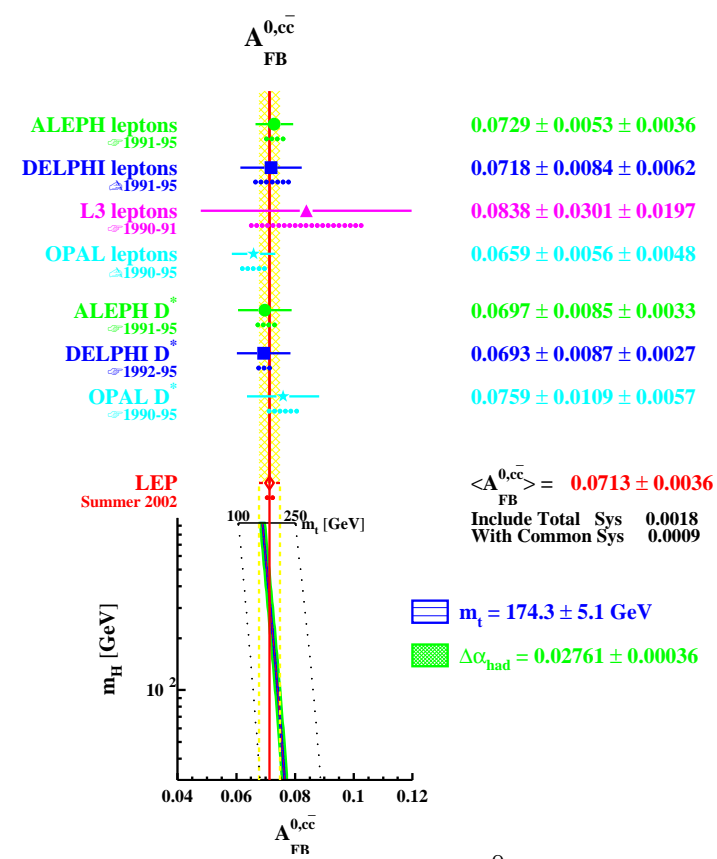

Figure 2. Measurements of $A_{\mathrm{FB}}^{\mathrm{c}, 0}$, the c quark asymmetry at the $\mathrm{Z}^{0}$ pole.

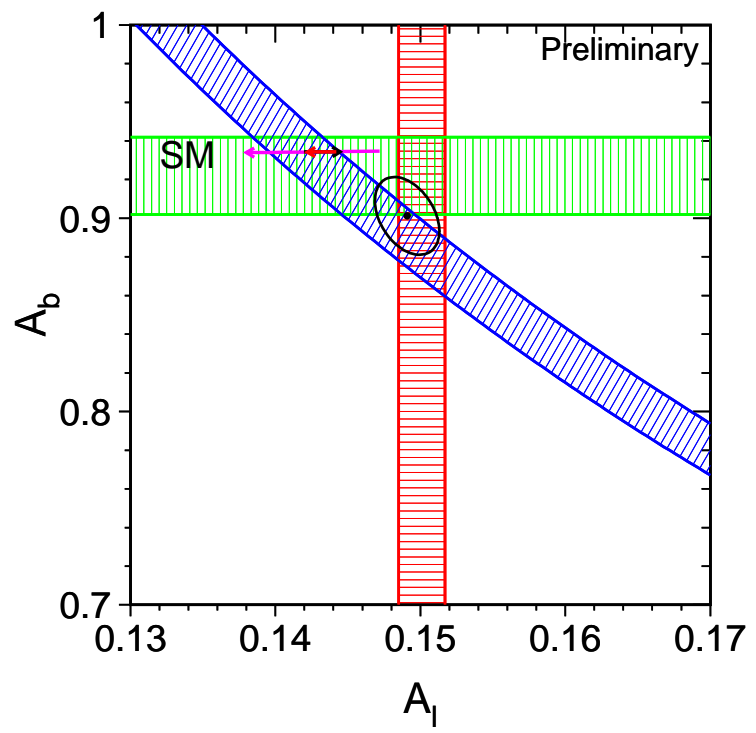

Figure 3. Combined results for the coupling parameters $A_{\mathrm{e}}\left(\equiv A_{\mathrm{l}}\right)$ and $A_{\mathrm{b}}$, and the asymmetry $A_{\mathrm{FB}}^{\mathrm{b}, 0}$. The bands show the \pm 1 standard deviation uncertainties, and the Standard Model expectation is shown by the arrow, with $m_{\mathrm{t}}=$ $174.3 \pm 5.1 \mathrm{GeV}$ and $m_{H}=300_{-186}^{+700} \mathrm{GeV}$.

right asymmetry $\left(A_{\mathrm{LR}}\right)$ at SLD and leptonic observables at LEP. It can be seen that the LEP $A_{\mathrm{FB}}^{\mathrm{b}, 0}$ (the diagonal band) is consistent with the Standard Model expectation, as is the SLD $A_{\mathrm{b}}$ measurement of $0.922 \pm 0.020$ [9]. However, using the measured $A_{\mathrm{e}}$ (which is somewhat above the Standard Model expectation) to extract $A_{\mathrm{b}}$ from $A_{\mathrm{FB}}^{\mathrm{b}, 0}$ results in a value of $A_{\mathrm{b}} 2.6$ standard deviations below the expected value. In contrast, the corresponding measurements for $A_{\mathrm{FB}}^{\mathrm{c}, 0}$ and $A_{\mathrm{C}}$ show good agreement with the Standard Model expectation.

A similar effect is visible on Figure 4, which shows the various asymmetry measurements interpreted within the Standard Model as determinations of the effective electroweak mixing angle for leptons $\sin ^{2} \theta_{\mathrm{W}}^{\text {eff }}$. The overall average from all measurements is

$\sin ^{2} \theta_{\mathrm{W}}^{\mathrm{eff}}=0.23148 \pm 0.00017$

but the fit $\chi^{2}$ is poor, at 10.2 for 5 degrees of freedom. This is entirely driven by the 2.9 stan- 


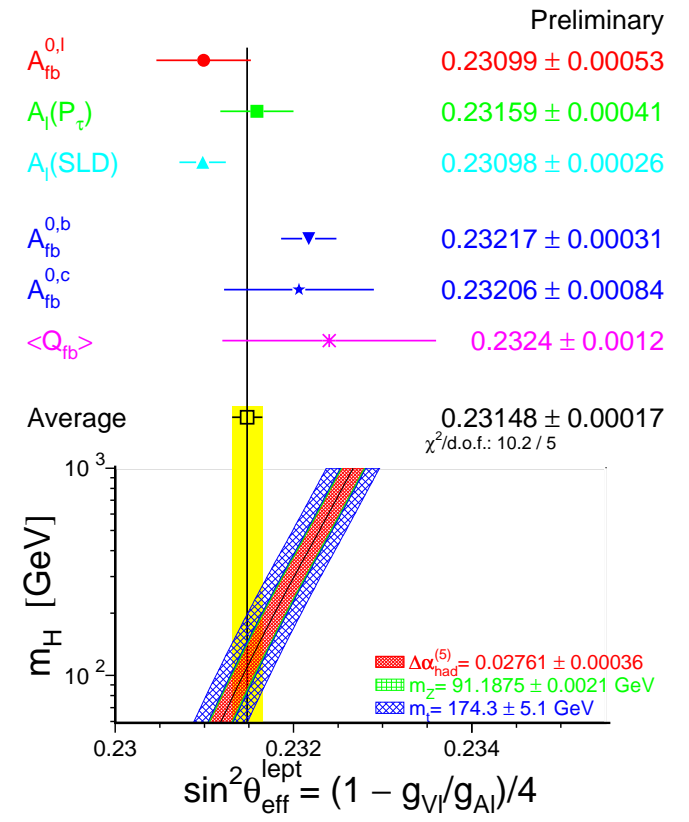

Figure 4. Determinations of the effective electroweak mixing angle $\sin ^{2} \theta_{\mathrm{W}}^{\text {eff }}$.

dard deviation discrepancy between $\sin ^{2} \theta_{\mathrm{W}}^{\text {eff }}$ measured from $A_{\mathrm{LR}}$ and $A_{\mathrm{FB}}^{\mathrm{b}, 0}$. The effect on the fitted Higgs mass is also shown $-A_{\mathrm{FB}}^{\mathrm{b}, 0}$ prefers a mass of around $400 \mathrm{GeV}$, whilst $A_{\mathrm{LR}}$ prefers a mass below $100 \mathrm{GeV}$.

\section{Discussion}

The LEP and SLD heavy flavour electroweak measurements have continued to improve over the last few years, with new improved analyses getting the maximum possible information from the data. However, there is now little scope for further reduction in the uncertainties, and almost all analyses have been finalised. Motivated by the three standard deviation discrepancy between $A_{\mathrm{LR}}$ and $A_{\mathrm{FB}}^{\mathrm{b}, 0}$, much effort has been invested in improving and checking the b quark asymmetry analyses in particular, where the uncertainty is still dominated by statistics. This discrepancy will most likely be left as a legacy from the LEP/SLD era - a statistical fluctuation, or perhaps a hint of new physics.

\section{Acknowledgements}

The author thanks the members of the LEP/SLD heavy flavour electroweak working group for the averages presented here and many useful discussions.

\section{REFERENCES}

1. G. Abbiendi et al., Phys. Lett. B546 (2002) 29.

2. DELPHI collab., DELPHI 2002-028 CONF 562.

3. K. Ackerstaff et al., Z. Phys. C75 (1997) 385.

4. The LEP collaborations, ALEPH, DELPHI, L3 and OPAL, Nucl. Instrum. Methods 378 (1996) 101.

5. A. Heister et al., Eur. Phys. J. C22 (2001) 201; DELPHI collaboration, DELPHI 2001048 CONF 476; M. Acciarri et al., Phys. Lett. B439 (1998) 225.

6. A. Heister et al., Eur. Phys. J. C24 (2002) 177; M. Acciarri et al., Phys. Lett. B448 (1999) 152; OPAL collab., OPAL Physics Note 226.

7. R. Barate et al., Phys. Lett. B434 (1998) 415; P. Abreu et al., Eur. Phys. J. C10 (1999) 219;

G. Alexander et al., Z. Phys. C73 (1996) 379.

8. R. Barate et al., Phys. Lett. B401 (1997) 163; P. Abreu et al., Eur. Phys. J. C10 (1999) 415; M. Acciarri et al., Eur. Phys. J. C13 (2000) 47; G. Abbiendi et al., Eur. Phys. J. C8 (1999) 217; K. Abe et al., Phys. Rev. Lett. 80 (1998) 660; R. Barate et al., Eur. Phys. J. C4 (1998) 557; R. Barate et al., Eur. Phys. J. C16 (2000) 597; P. Abreu et al., Eur. Phys. J. C12 (2000) 209; P. Abreu et al., Eur. Phys. J. C12 (2000) 225; K. Ackerstaff et al., Eur. Phys. J. C1 (1998) 439; G. Alexander et al., Z. Phys. C72 (1996) 1; SLD collab., contributed paper 174 to ICHEP98 Vancouver, Canada.

9. K. Abe et al., Phys. Rev. Lett. 88 (2002) 151801. 